\title{
Efecto de bioplaguicidas sobre la incidencia de plagas y enferme- dades foliares y componentes de rendimiento del frijol común (Phaseolus vulgaris L.) en Santa Rosa de Copán
}

Kevin Jarod Mejía ${ }^{1}$

\section{RESUMEN}

El daño causado por plagas y enfermedades es uno de los principales factores que afectan la producción a nivel nacional del frijol ( $P$. vulgaris) ya que atacan todos los órganos y etapas de crecimiento, producción y almacén. Con objetivo de evaluar el efecto de bioplaguicidas sobre la incidencia de plagas y enfermedades foliares en frijol común ( $P$. vulgaris) en Santa Rosa de Copán durante la campaña de enero-abril de 2017, se realizaron dos ensayos. El primero consistió en evaluar el control de los bioplaguicidas sobre la incidencia de plagas; y en el segundo se valoró la incidencia y severidad de enfermedades foliares, ambos casos se contrastaron bioplaguicidas con un testigo químico y uno absoluto, la aplicación de los mismos se realizó en las fases V4 (vegetativa) y R5 (regenerativa, prefloración). Las variables consideradas estuvieron encaminadas a evaluar las plagas, las enfermedades más comunes que aparecieron en campo, y las variables de rendimiento. Los resultados reflejaron diferencia estadística significativa con respecto a $\mathrm{P} \leq 0.05$, donde las plagas con mayor frecuencia fueron Salta hojas (Empoasca kraemeri), Mosca blanca (Bemishia tabaci), Diabróticas (Diabrotica balteata), a la vez se registró presencia de controladores biológicos como fue Orius insidosus; las enfermedades con mayor presencia fueron Mancha angular (Phaseoisariopsis griseola) y Roya (Uromyces appendiculatus). En las variables de rendimiento se encontró diferencia estadística significativa con respecto al testigo absoluto. En base a los resultados obtenidos en ambos ensayos se recomienda usar Engeo 24.7 SC a 1L.ha-1 y Amistar xtra 28 SC a misma dosis de forma preventiva.

Palabras clave: Bioplaguicidas, plagas, enfermedades, frijol.

${ }^{1}$ Beneficiario de una beca básica de la DICYP. Profesor del Departamento de Producción vegetal, Centro Regional Universitario de Occidente (UNAH-CUROC): maskmejia@gmail.com 


\section{ABSTRACT}

The damage caused by pests and diseases is one of the factors that affect the national production of beans ( $P$. vulgaris) as they attack all organs and stages of growth, production and storage; In order to evaluate the effect of biopesticides on the incidence of pests and foliar diseases in common bean ( $P$. vulgaris) in Santa Rosa de Copán during the January-April 2017, two trials were carried out: the first consisted of evaluating the control of biopesticides on the incidence of pests; and in the second, the incidence and severity of foliar diseases were evaluated, both cases were contrasted biopesticides with a chemical chemical and an absolute, the application of them was carried out in the phases V4 (vegetative) and R5 (regenerative, pre-flowering). The variables considered are aimed at evaluating pests, the most common diseases that appeared in the field, and performance variables. The results differ significantly compared to P0.05, where the leaves with more frequency were Salta leaves (Empoasca kraemeri), White fly (Bemishia tabaci), Diabrotica (Diabrotica balteata), at the same time with the presence of biological controllers such as Orius insidosus; The diseases with greater presence were Angular spot (Phaseoisariopsis griseola) and Roya (Uromyces appendiculatus). In the performance variables there were significant differences with respect to the absolute control. Engeo 24.7 SC to 1L.ha-1 and Amis$\operatorname{tar}$ xtra $28 \mathrm{SC}$ at the same dose in a preventive manner.

Keywords: Biopesticides, pests, diseases, beans. 


\section{INTRODUCCIÓN}

En Honduras, el cultivo de frijol (Phaseolus vulgaris L.) ocupa el segundo lugar de producción de granos básicos y representa el 3\% del PIB agropecuario (CEPAL 2011). Además representa el $20.25 \%$ del área dedicada a cultivos de consumo interno, y anualmente se producen 88,700 toneladas, con rendimientos de 0.9 toneladas/hectárea (CEPAL 2011). El frijol es la fuente principal de proteínas para la mayoría de la población rural y urbana de escasos recursos de Centro América y El Caribe (Rosas 2000, tomado de Campoverde, 2004). En la región de occidente, el cultivo está en manos de pequeños agricultores, los cuales tienen limitado acceso a insumos, tierras fértiles y mecanización (SAG, 2014).

Dentro del grupo de las leguminosas que poseen semillas comestibles, el frijol común corresponde a una de las más importantes. Actualmente se encuentra distribuido en los cinco continentes y es un componente esencial de la dieta, especialmente en Centroamérica y Sudamérica (Ulloa et al., 2011).

El daño causado por plagas y enfermedades es uno de los principales factores que afectan la producción a nivel nacional del frijol ( $P$. vulgaris) ya que atacan todos los órganos y etapas de crecimiento, producción y almacén. Causando daños directos a la productividad, independientemente del sistema de producción y tecnología que se trate (Sagarpa, 2016).

En la actualidad los bioplaguicidas se han convertido una opción para el control biológico de plagas y enfermedades de los cultivos (Nava-Pérez et al, 2012), no obstante, son productos que requieren de evaluar y comprobar su efectividad en todos los agroecosistemas. Se considera estos productos no muy conocidos por los productores con respecto en su efectividad en el control de plagas y enfermedades, razón por que actualmente no se cuenta con muchas experiencias prácticas e investigativas. Particularmente en Santa Rosa de Copán los pequeños productores de granos básicos no emplean productos químicos o bien usan los incorrectos.

El objetivo de la investigación consistió en evaluar el efecto de bioplaguicidas sobre la incidencia de plagas y enfermedades foliares en frijol común (Phaseolus vulgaris L.) en condiciones de producción en Santa Rosa de Copán durante la campaña de enero-abril de 2017. 


\section{MATERIALES Y MÉTODOS}

\section{Descripción del área de estudio}

El estudio se realizó en la finca "El Padre" de la comunidad "La Montañita" ubicada a $12 \mathrm{~km}$ de Santa Rosa de Copán (S.R.C), cabecera departamental de Copán en la región occidental de Honduras. El agroecosistema cuenta con un área total de 5.6 hectáreas, la temperatura media anual es de $24^{\circ} \mathrm{C}$ y su precipitación media anuales es de $1100 \mathrm{~mm}$, ello se corresponde al bosque seco tropical (bs-T (c)) según la clasificación de zonas de vida de Holdridge (2000). En este predio existe un predominio de relieve ondulado con pendientes superiores al $20 \%$.

\section{Ensayos y variables medidas}

El trabajo consistió en dos ensayos de cuatro tratamientos cada uno (Cuadro 1), cuya finalidad consistió en medir el efecto de los bioplaguicidas sobre las plagas y las enfermedades.

\section{Cuadro 1. Ensayos y tratamientos para la evaluación de plagas y enfermedades} foliares sobre frijol común (P. vulgaris)

\begin{tabular}{|c|c|c|}
\hline No. & $\begin{array}{c}\text { Ensayo 1: evaluación de } \\
\text { bioplaguicidas sobre la incidencia } \\
\text { de plagas foliares }\end{array}$ & $\begin{array}{l}\text { Ensayo 2: Evaluación de } \\
\text { bioplaguicidas sobre la incidencia de } \\
\text { enfermedades foliares }\end{array}$ \\
\hline 1 & Control absoluto & Control absoluto \\
\hline 2 & Beauberia basiana a 240g.ha-1 & Basillus subtilis a 1L.ha-1 \\
\hline 3 & Metarrizium anisopleae a 240g.ha-1 & Trichoderma harzianum a 240g.ha-1 \\
\hline 4 & Engeo 24.7 SC a 1L.ha-1 & Amistar xtra $28 \mathrm{SC}$ a $1 \mathrm{~L}^{2}$ ha-1 \\
\hline
\end{tabular}

La aplicación de los productos se realizó a los 25 y 35 DDS en V4 y R5 respectivamente. Fuente: Elaboración propia

Los muestreos se realizaron de manera sostenida después de la emergencia del cultivo a los 8 días después de sembrado, manteniéndose de forma sostenida hasta la cosecha. Las fases que se consideraron críticas para la evaluación en ambos ensayos durante V3 (primera hoja trifoliada completamente abierta), V4 (tercera hoja trifoliada completamente abierta) como fases vegetativas y, R5 (prefloración o botón floral), R6 (floración completamente abierta), y R7 (formación de las primeras vainas) como fases regenerativas. 
La toma de muestra consistió en tomar 10 plantas de forma aleatoria por cada tratamiento, tanto las plagas como las enfermedades en las hojas y vainas de la planta fueron colocadas en bolsa tipo ziploc para su posterior identificación en laboratorio. Las variables que se consideraron para cada ensayo fueron las siguientes:

\section{Ensayo 1: Monitoreo de plagas más frecuentes}

- El conteo de plagas presentes en el cultivo durante las fases vegetativas $(V)$ y regenerativas (R) desde V3, V4, R5, R6 hasta R7.

\section{Ensayo 2: Incidencia y severidad de enfermedades foliares}

La medición de lesiones ocasionadas por enfermedades se realizó durante las fases vegetativas (V) y regenerativas (R) desde V3, V4, R5, R6 hasta R7.

Las mediciones del rendimiento para ambos ensayos consistió en: número de legumbre por planta $(L x P)$, número de grano por legumbre $(G x L)$, y no. de grano dañado por legumbre (GDxL).

\section{Diseño y arreglo experimental}

El diseño experimental empleado para los ensayos fue en bloque completamente al azar (BCA) con cuatro tratamientos cada ensayo y replicado cuatro veces para un total de 16 unidades experimentales por cada ensayo. La unidad experimental (UE) consistió en parcelas de $12.8 \mathrm{~m} 2$ (4 hileras a $0.8 \mathrm{~m}$ y $4 \mathrm{~m}$ de longitud). Para reducir el error del experimento por el efecto de borde, en la toma de datos y la cosecha, se recortaron las hileras centrales a $3 \mathrm{~m}$.

\section{Labores agrotécnicas}

Las labores agrotecnicas al suelo y cultivo se realizaron de forma convencional a la que realiza el productor. La fertilización fue granulada al suelo con DAP 18-46-0 a la siembra, urea $46 \%$ y $\mathrm{KCl} 60 \%$ a los 25 y 35 días después de sembrado.

\section{Análisis estadístico}

Para contrastar la hipótesis nula de que ningún bioplaguicida tiene diferencia significativa sobre la incidencia y severidad de plagas y enfermedades foliares en el frijol común se hizo un análisis de varianza (One-Way ANOVA) para los ensayos y una 
separación de medias corriendo la prueba de Duncan con un margen de error del $5 \%$. El análisis de los datos se realizó con el software estadístico SPSS ver. 21 corrido en Machintoch.

\section{RESULTADOS}

\section{Ensayo 1: Evaluación de bioplaguicidas sobre la incidencia de plagas foliares.}

Durante el desarrollo del ensayo se encontraron tres plagas con mayor presencia las cuales fueron salta hojas (Empoasca kraemeri), Mosca blanca (Bemishia tabaci), Diabrótica (Diabrotica balteata) (Figura 1), a la vez se encontró la presencia de Orius (Orius insidiosus) como controlador biológico, el cual es un depredador de áfidos (Aphis spp.).

Figura 1. Principales plagas encontradas en el ensayo
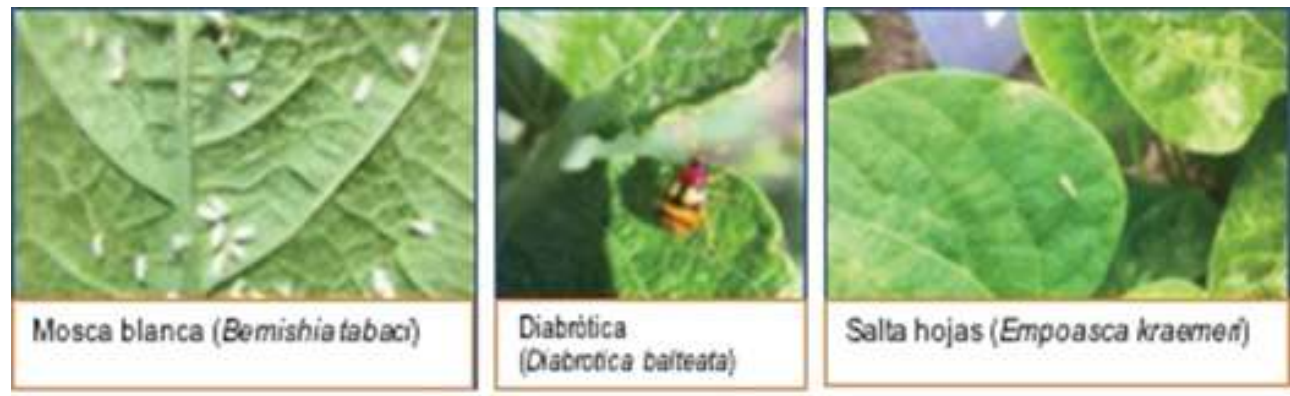

Fuente: Elaboración propia

\section{Salta hojas (Empoasca kraemeri)}

La figura 2 muestra el comportamiento poblacional por Salta hojas (Empoasca kraemeri) en el cultivo de frijol (P. vulgaris L.) durante las fases de desarrollo del cultivo que se consideraron para este ensayo. En la figura se observa como las poblaciones de insectos se mantiene estable (por debajo del umbral) luego de la aplicación de los productos que se realizó en las fases vegetativas del cultivo V3 y V4 (Prefloración). Después de floración la población se incrementa, sin embargo en esta fase de desarrollo de la planta el daño al fruto es mínimo. El tratamiento que mostró mayor número de salta hojas fue el control absoluto y el Metazam, siendo más efectivo para el ensayo el uso del Engeo (control químico) que durante todo el ciclo mantuvo poblaciones bajas de la plaga. 
Figura 2. Comportamiento de salta hojas (Empoasca kraemeri) al tratamiento con Metazam, Bazam (biológicos), al testigo químico y testigo absoluto.
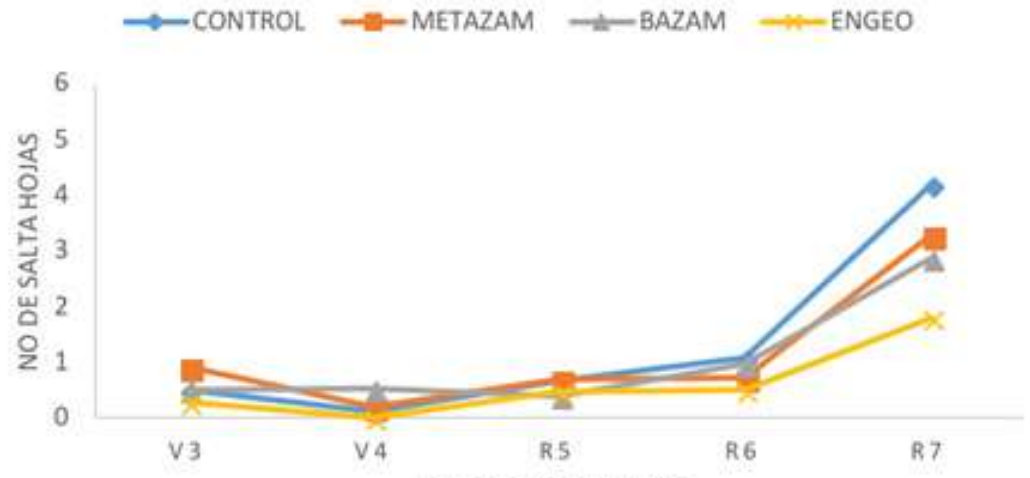

Fuente: Elaboración propia

Tanto los tratamientos con biológico y químico lograron mantener las poblaciones de Lorito verde estables durante las dos aplicaciones que se realizó previo a la floración por lo que no se afectó la formación del fruto en su etapa más joven.

\section{Mosca blanca (Bemishia tabaci)}

En la figura 3 se observa una disminución de la aparición de mosca blanca (Bemishia tabaci) después de la primera y segunda aplicación (V3 y V4 respectivamente), al igual que en la plaga anterior se muestra un leve incremento de la misma al inicio de la floración de la planta, sin embargo, el daño en la planta, particularmente a la vaina es nulo.

Figura 3. Efecto de bioplaguicidas sobre la presencia de mosca blanca (Bemishia tabaci) en frijol común ( $P$. vulgaris $L$.)

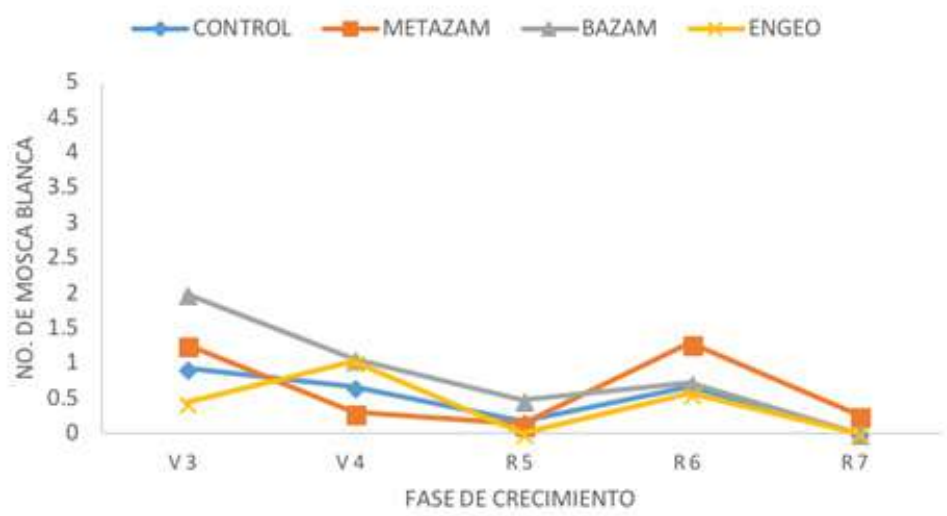

Fuente: Elaboración propia 
Considerando que la floración es un periodo crítico para la planta porque es cuando inicia con la formación del fruto (vaina), el incremento de mosca blanca no afectó la formación de las mismas, es importante considerar que la plaga a este punto el daño que causa es principalmente en las hojas, no obstante, la planta mostró ser tolerante.

\section{Diabróticas (Diabrotica balteata)}

En la figura 4 se observa que durante de la fase V3 a V4 el número de diabróticas (Diabrotica balteata) aumenta tanto en los tratamientos con bioplaguicidas (Bazam y Metazam) como en el control absoluto, en R5 (Floración) vuelve a disminuir su presencia, sin embargo, en el tratamiento con químico (Engeo 24.7 SC) la población de insectos se mantuvo en 0. El Engeo 24.7 SC al ser un producto sistémico se mantiene durante más tiempo dentro de la planta, por lo que al morder la hoja el insecto muere.

Figura 4. Efecto de bioplaguicidas sobre la presencia de diabróticas (Diabrotica balteata) en plantas de frijol común ( $P$. vulgaris)

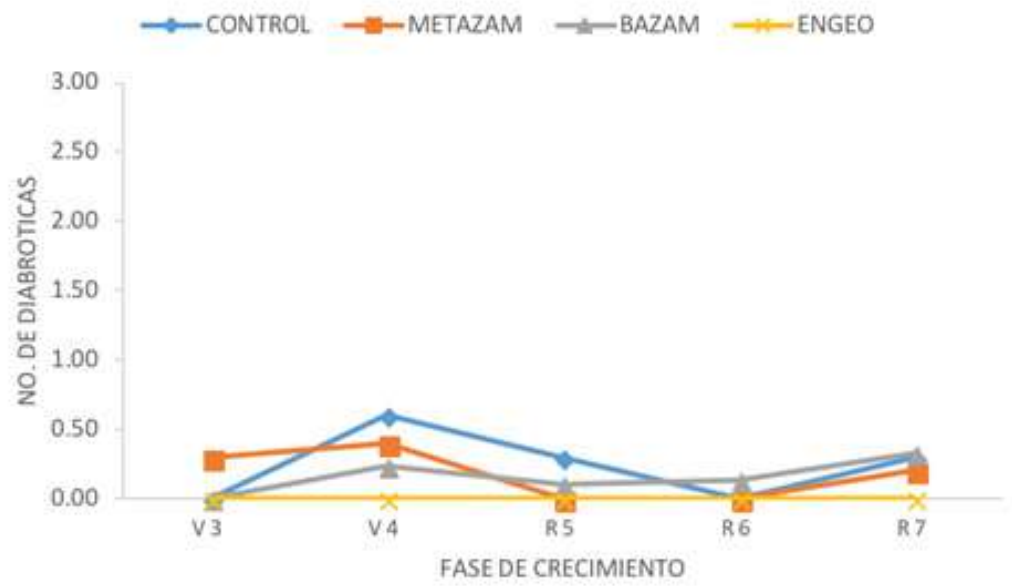

Fuente: Eladoracion propla

\section{Plagas en vaina de la planta}

De las plagas más comunes que afectan la vaina de la planta del frijol como son picudo de la vaina (Apion godmani) y el chinche del frijol o chinche hedionda (Nezara viridula) siendo los registros de estas a una chinche por planta, no obstante, es de tener especial cuidado de esta plaga por el daño que ocasiona al grano cuando está pequeño (recién formado), porque una vez afectado no se desarrolla (Figura 5). 
Figura 5. Plagas de la vaina encontradas en el frijol

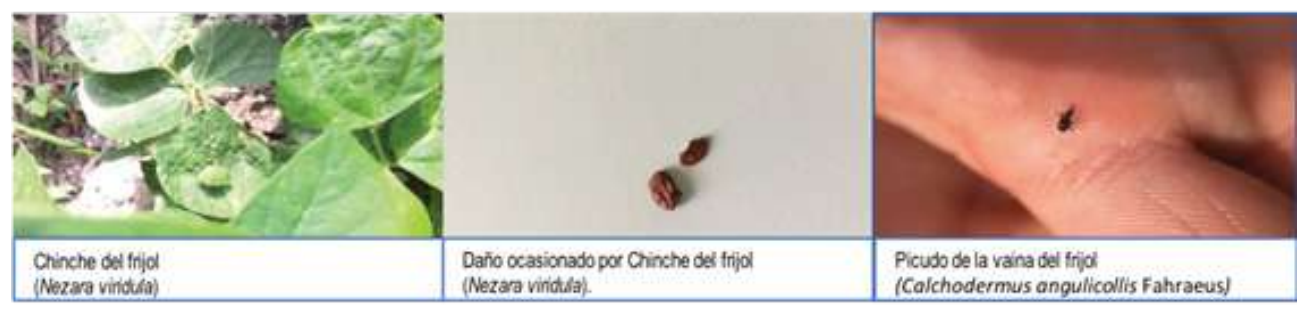

Fuente: Elaboración propia

\section{Insectos benéficos}

Orius (Orius insidiosus)

Uno de los controladores biológicos que apareció en el ensayo fue el Orius (Orius insidiosus). Los registros de este insecto se empezaron a manifestar a partir de R5 (post-floración) en el cual se encontró un incremento para R7 en el tratamiento con Metazam y Bazam superando los 2.4 y 1.5 individuos por planta respectivamente, mientras tanto en el control químico con Engeo los registros no fueron superiores a un individuo por planta. Otro insecto benéfico que se encontró en los ensayos fue de Cycloneda spp. (Figura 6). Esta especie al igual que Orius es un depredador de áfidos (Aphis spp.), sin embargo, las poblaciones fueron muy bajas, por esta razón no fueron contabilizadas para el ensayo. La reducción de Orius por el empleo del control químico (Engeo) refleja su incidencia en la población de individuos benéficos para el cultivo.

Figura 6. Presencia de Cycloneda spp (izq). y Orius insidiosus (der) en plantas de frijol

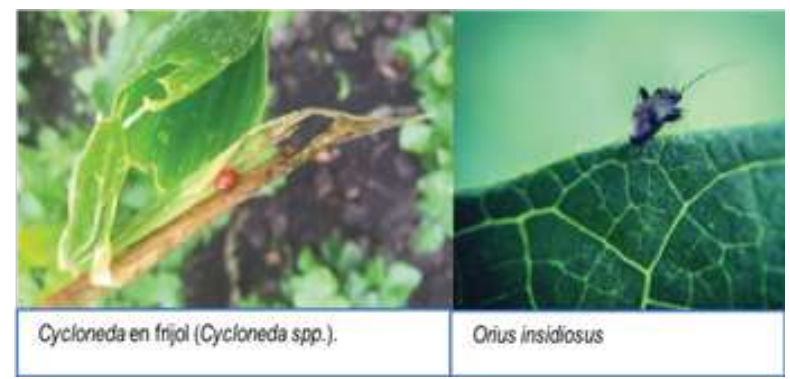

Fuente: Elaboración propia 


\section{Rendimiento}

Una vez finalizado el ciclo del cultivo se contabilizaron datos de rendimiento siendo: Número. de vaina por planta (V/P). Número de grano por vaina $(G / V)$. Número de grano dañado por legumbre (GD/L) y el Peso de granos por planta $(\mathrm{g})$.

En la figura 7 se observa que en las mediciones de vaina/Planta existe diferencia significativa de acuerdo con la prueba de Duncan, siendo el insecticida Engeo con mayor número de vainas, de igual forma existe diferencia estadística para el peso en granos por planta donde se encuentra una diferencia superior a $6 \mathrm{~g}$ por parte del tratamiento con Engeo con respecto al control.

Figura 7. Efecto de bioplaguicidas insectiles sobre variables de rendimiento en plantas de frijol común. *Letras distintas significan promedios distintos para Duncan a una $\mathrm{P} \leq 0.05$.

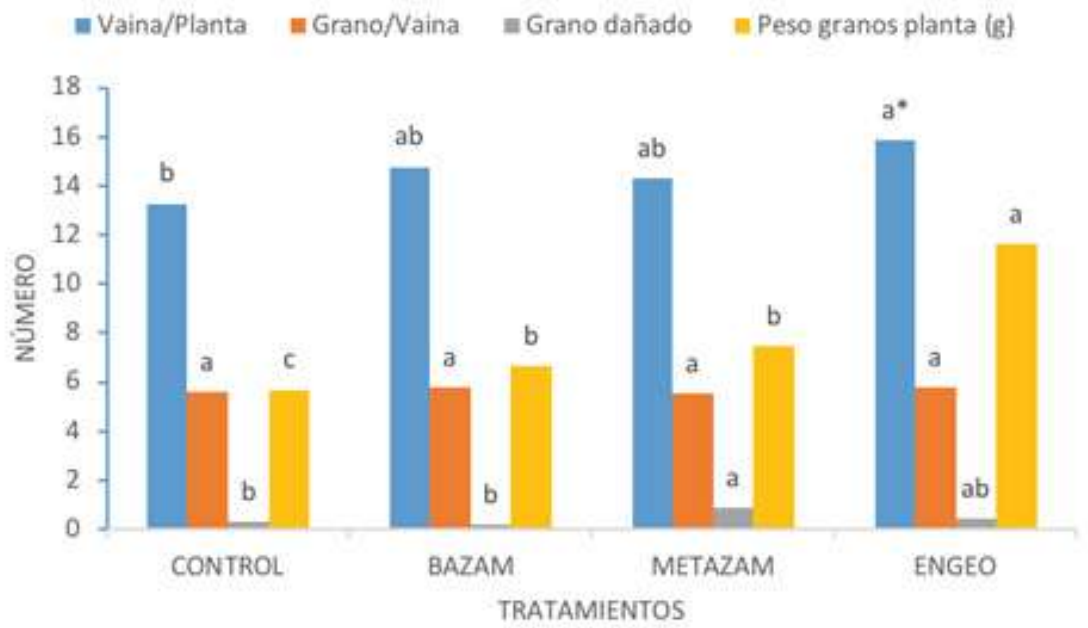

Fuente: Elaboración propia

El daño encontrado en los granos fue ocasionado por chinche verde (Nezara viridula), sin embargo, los resultados reflejaron que no es significativo. Aunque es recomendable reducir la afección por esta plaga, para evitar la reproducción del insecto en campo para futuras siembras. 
Ensayo 2: Evaluación de bioplaguicidas sobre la incidencia y severidad de enfermedades foliares.

En las evaluaciones que se realizaron en el cultivo en los cuatro tratamientos establecidos se encontró predominancia en lesiones ocasionadas por roya (Uromyces appendyculatus) y mancha angular (Phaseoisariopsis griseola). Los daños se manifestaron tanto en hojas como en vainas.

\section{Roya (Uromyces appendyculatus)}

La roya (Uromyces appendiculatus) es común a nivel mundial y se considera como uno de los problemas más importante que afectan la producción del frijol en Latinoamérica y el trópico en general (Zaumeyer y Meiners, 1975; Languidey y Aguilera, 1983).

Durante las cuatro fases del cultivo evaluadas V4, R5, R6 y R7 (Figura 8 y 9), el tratamiento con Amistar fue el que tuvo menos afección, manteniéndose por debajo del $20 \%$ durante el ciclo del cultivo, y para el cual se encontró diferencia estadística significativa con respecto al control absoluto, este último mostró un incremento considerable de lesión por roya (Uromyces appendiculatus) desde el desarrollo vegetativo prefloración.

Figura 8. Efecto de bioplaguicidas sobre la incidencia de roya (Uromyces appendiculatus) en plantas de frijol común.

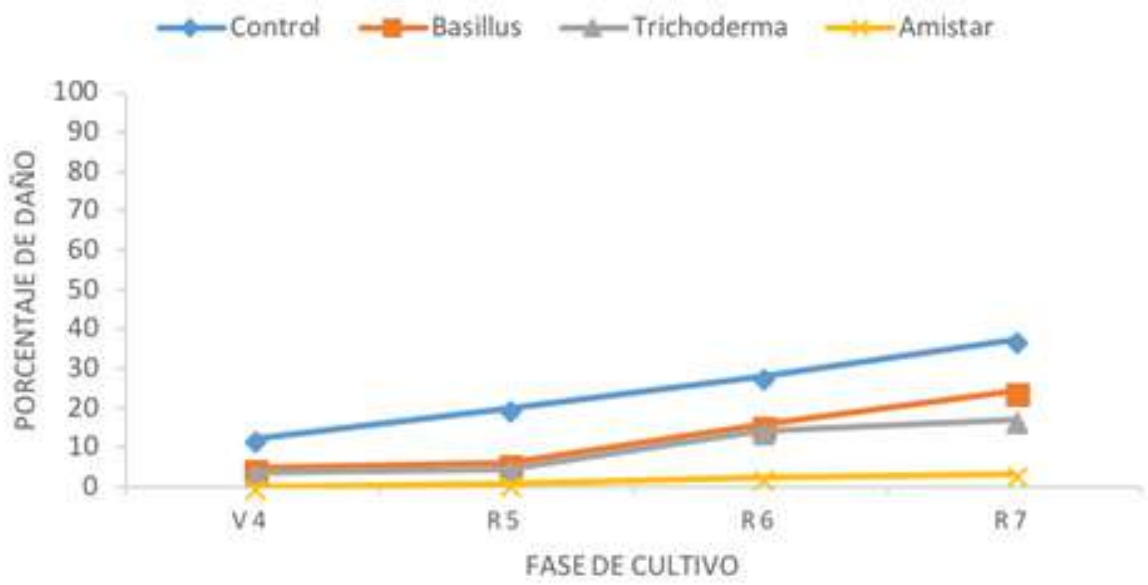

Fuente: Elaboración propia 
La reducción del rendimiento que causa la roya del frijol se debe a los disturbios fisiológicos en la planta y la destrucción del área foliar (Gálvez; Galindo; Álvarez, 1977). Con base en lo anterior se añade la gran cantidad de esporas que el hongo produce rápida y permanentemente (CIAT, 1980).

Figura 9. Daño ocasionado por roya (Uromyces appendiculatus) ocasionada en la hoja de planta de frijol (P. vulgaris)
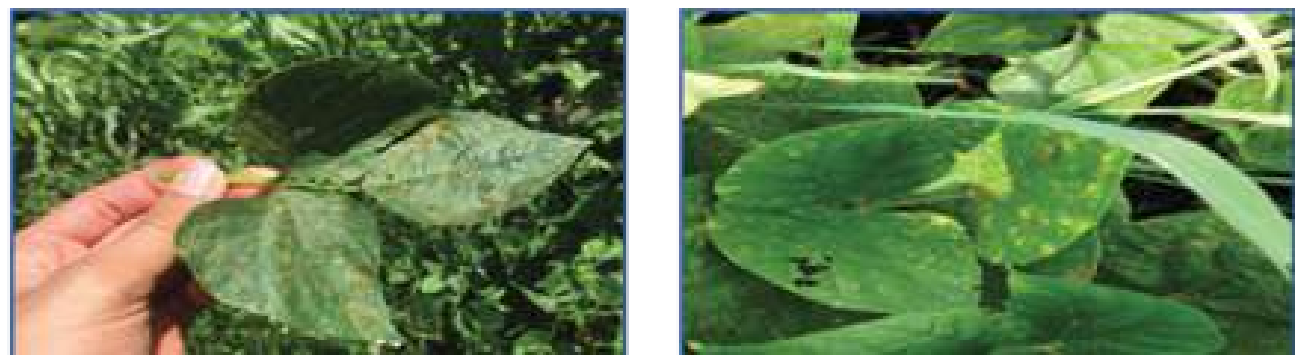

Fuente: elaboración propia

\section{Mancha angular (Phaseoisariopsis griseola)}

La mancha angular (Figura 10 ) es una de las enfermedades que mayor severidad generó sobre el cultivo de frijol común ( $P$. vulgaris). En la figura 11 se observó que el tratamiento con Amistar fue lo que menos problemas presentó, durante las cuatro fases evaluadas. Los resultados mostraron diferencia significativa entre tratamiento en las cuatro fases del cultivo que se evaluó, siendo el tratamiento con Amistar el que menos daño presentó, seguido de Trichoderma (Trichoderma harzianum).

Figura 10. Daño ocasionado por Mancha angular (Phaseoisariopsis griseola) en la hoja de la planta de frijol. (A). Problema de MA en envés de hoja. (B) Problema de MA y Roya
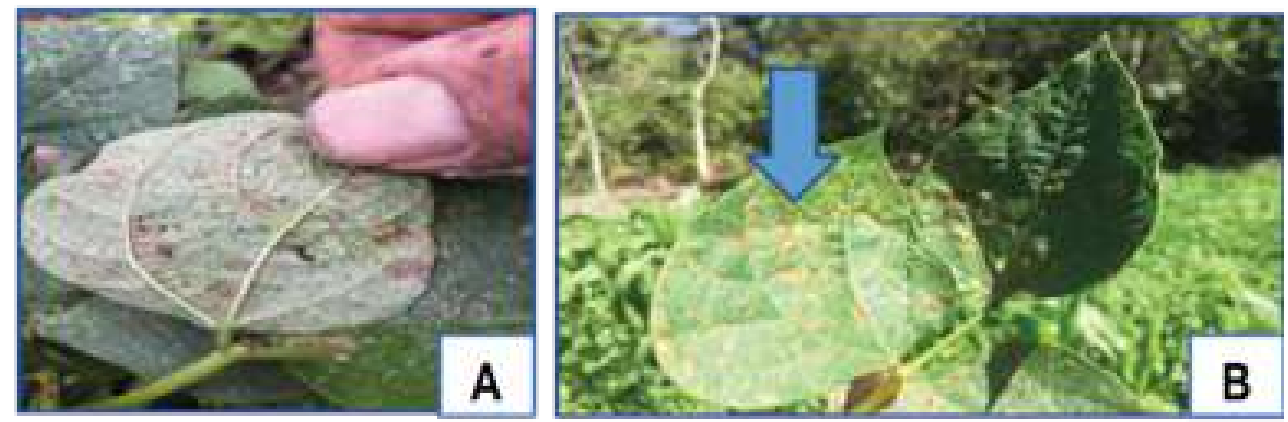

Fuente: elaboración propia 
Figura 11. Efecto de bioplaguicidas sobre la incidencia de Mancha angular (Phaseoisariopsis griseola) en plantas de frijol común

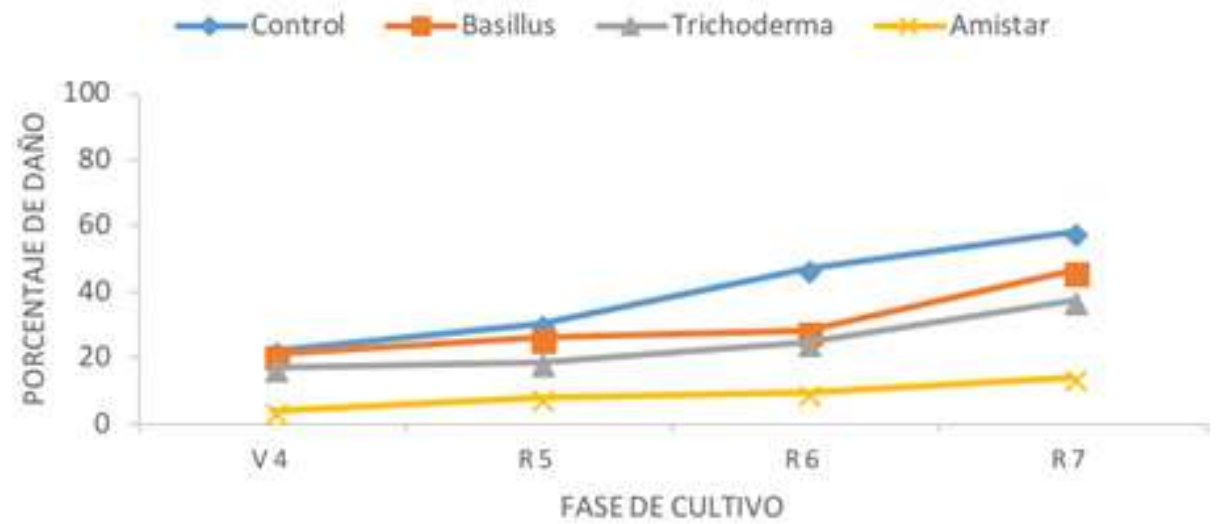

Fuente: elaboración propia

En este estudio se usó la variedad Amadeus-77, misma que presenta tolerancia a enfermedades como roya y mancha angular, no obstante, se nota en el tratamiento de control absoluto que la afección tuvo un crecimiento más acelerado una vez que la planta alcanzó la floración.

\section{Mancha angular en la vaina de la planta}

De las plantas evaluadas en R8 (fase de maduración del fruto) se encontró que todas las vainas presentaron lesiones ocasionadas por mancha angular (Figura 12). Los resultados mostraron diferencia significativa con respecto a Duncan, donde las mayores afecciones se encontraron en el control con un 21\% (equivalente a un cuarto de vaina), mientras que los tratamientos con Basillus y Trichoderma mostraron afecciones leves por debajo del $12 \%$ y $9 \%$ respectivamente. Mientras que el tratamiento con Amistar no presentó mayores síntomas, lo cual no generó daños considerables con respecto al control absoluto.

\section{Rendimientos}

En la figura 13 muestra que se encontró diferencia estadística significativa en las evaluaciones de vaina/planta y peso de granos por planta, siendo el tratamiento con amistar el que registró mejores resultados para ambas variables, mientras que en el número de granos por vaina (Grano/Vaina) y grano dañado (Grano/Daño) no se encontró diferencia estadística con respecto a Duncan. 
Figura 12. Efecto de bioplaguicidas sobre la incidencia de Mancha angular (Phaseoisariopsis griseola) en vainas de frijol común. *Letras distintas significan promedios distintos para Duncan a una $\mathrm{P} \leq 0.05$

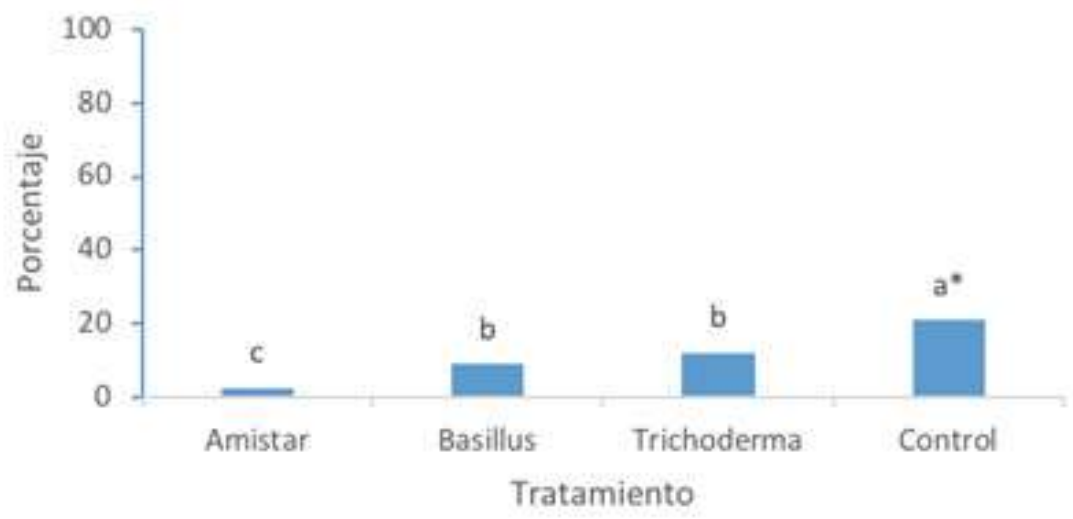

Fuente: elaboración propia

Figura 13. Efecto de bioplaguicidas fúngicos sobre variables de rendimiento en frijol común. *Letras distintas significan promedios distintos para Duncan a una $\mathrm{P} \leq 0.05$.

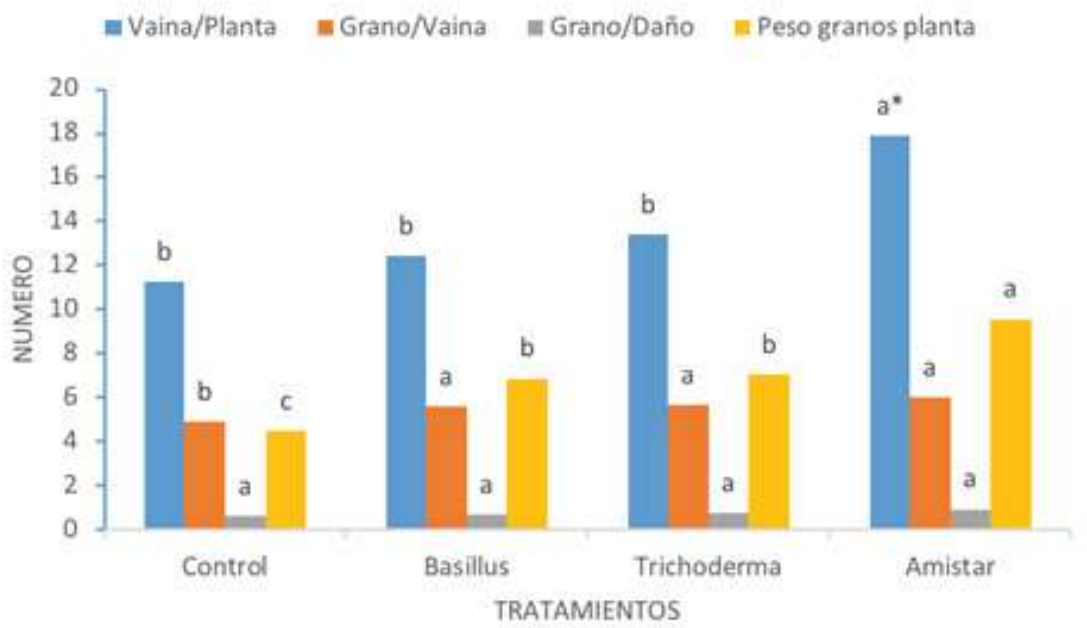

Fuente: elaboración propia 


\section{CONCLUSIONES}

Las plagas que mayormente incidieron en las hojas del frijol común (P. vulgaris $L$.) fueron Salta hojas (Empoasca kraemeri), mosca blanca (Bemishia tabaci) y diabróticas (Diabrotica balteata). Mientras que en la vaina se registró afecciones ocasionadas por Chinche del frijol o chinche hedionda (Nezara viridula).

Las enfermedades más incidentes en las condiciones de estudio fueron roya (Uromyces appendiculatus) y Mancha angular (Phaseoisariopsis griseola). Los controles con Engeo 24.7 SC a 1L.ha-1 y Amistar xtra 28 SC a 1 L.ha-1 resultaron ser el más efectivos para el control de las plagas y enfermedades.

\section{RECOMENDACIONES}

1. Replicar el ensayo en otras épocas productivas del año para contrastar resultados.

2. Utilizar otras variedades de frijol tanto locales como mejoradas.

3. Medir el comportamiento de plagas y enfermedades en variedades tanto mejoradas como criollas para identificar la respuesta de ambos.

4. Emplear varias dosis de biológicos para comprobar su efectividad en el control de plagas y la reducción por afección de enfermedades.

\section{AGRADECIMIENTOS}

Un especial agradecimiento a la Dirección de investigación Científica y de Posgrado (DICyP) y a la Finca "El Padre" por el apoyo económico y logístico para llevar a cabo esta investigación.

\section{REFERENCIAS BIBLIOGRÁFICAS}

Campoverde, D. (2004). Respuesta de tres variedades de frijol a tres poblaciones y dos niveles de nitrógeno. Proyecto especial presentado como requisito parcial para optar al título de Ingeniero Agrónomo en el grado Académico de Licenciatura. Zamorano, 
Honduras 34 .

Centro Internacional de Agricultura Tropical (CIAT). (1980). La roya del frijol y su control. Guía de estudio Serie 04 5B- 06.06. Cali. Colombia. 5-17

Centro Internacional de Agricultura Tropical (CIAT). (2010). Guía para la identificación de plagas y enfermedades en frijol. Pan-Africa Bean Research Alliance Cali. Colombia. 67 CEPAL (Comisión Económica para América Latina y el Caribe, sede subregional en México). (2011). Subregión norte de América Latina y el Caribe: información del sector agropecuario, 2000-2010. CEPAL. 69

Nava-Pérez, Eusebio (2012). Bioplaguicidas: una opción para el control biológico de plagas. (U. A. México., Ed.) Ra Ximhai. 17-29

Holdridge, L. (2000). Ecología basada en Zonas de Vida. Instituto Interamericano de Cooperación para la Agricultura (Vol. 83). San José, Costa Rica: Colección Libros y Materiales Educativos/IICA.

Ulloa, José Armando (2011). El frijol (Phaseolus vulgaris): su importancia nutricional y como fuente de fitoquímicos. Fuente, 5-9.

Sagarpa. (2016). Manual de plagas y enfermedades en frijol. Campaña manejo fitosanitario del frijol. Comité estatal de Sanidad vegetal de Guanajuato. A.C. 1- 24

SAG. (2014). Producción de granos básicos en Santa Rosa de Copán y Honduras. Charla dictada por José Aparicio Ortega. 11 de marzo de 2014 en Centro Universitario Regional de Occidente.

Zaumeyer, J; Meiners, J. (1975). Disease resistance in beans. Ann. Rev. Phytopath. 13: 320322.

Languidey, P.; Aguilera, M. (1983). Control químico de la roya (agente causal: Uromyces phaseoli (Rebe) Wint) del frijol (Phaseolus vulgaris L.). Rev. Boliviana. Inv. 1:95-97 